\title{
Fine-Scale Plasticity of Microscopic Saccades
}

\author{
Katharina Havermann, ${ }^{1,2}$ Claudia Cherici, ${ }^{3}$ Michele Rucci, ${ }^{3 *}$ and Markus Lappe ${ }^{1,2 *}$ \\ ${ }^{1}$ Institute for Psychology, University of Muenster, 48149 Muenster, Germany, ${ }^{2}$ Otto Creutzfeldt Center for Cognitive and Behavioral Neuroscience, \\ University of Muenster, 48149 Muenster, Germany, and ${ }^{3}$ Department of Psychology and Graduate Program in Neuroscience, Boston University, Boston, \\ Massachusetts 02215
}

When asked to maintain their gaze steady on a given location, humans continually perform microscopic eye movements, including fast gaze shifts known as microsaccades. It has long been speculated that these movements may contribute to the maintenance of fixation, but evidence has remained contradictory. We used a miniaturized version of saccadic adaptation, an experimental procedure by which motor control of saccades is modified through intrasaccadic displacements of the target. We found that the statistical distribution of microsaccade amplitudes changes after brief exposure to systematic shifts of the fixation point during microsaccade occurrence. Shifts in the same directions as microsaccades produce movements with larger amplitudes, whereas shifts against microsaccade directions result in smaller movements. Our findings show that microsaccades are precisely monitored during fixation and that their motor program is modified if the postsaccadic target position is not at the expected retinal location. These results demonstrate that saccadic adaptation occurs even when the stimulus is already close to the foveal center and precise execution of the movement may not be critical. They support the proposal that adaptation is necessary to maintain a consistent relationship between motor control and its visual consequences and that the representation of space is intrinsically multimodal, even during fixation.

Key words: fixation; learning; microsaccades; saccadic adaptation

\section{Introduction}

Humans and many other species rely on fast eye movements (saccades) to relocate the high-acuity region of the retina and acquire information from a visual scene. The precision of these gaze shifts is continually monitored by the oculomotor system, and saccades are adjusted if they no longer land at their intended locations (McLaughlin, 1967; Pélisson et al., 2010). This adaptation process is believed necessary to ensure that saccades accurately move the eye to enable examination of their targets with the high-acuity fovea.

However, microscopic saccades, known as microsaccades, also occur when the target of interest already falls within the foveal region of the retina (Barlow, 1952; Zuber et al., 1965; Steinman et al., 1973). Humans perform frequently these movements even when looking at a single point, and their purpose has been fiercely debated (Collewijn and Kowler, 2008; Rolfs, 2009). Microsaccades have been commonly regarded as largely "involuntary" movements necessary for preventing neural adaptation and the perceptual fading experienced in the complete absence of retinal image motion (Ditchburn et al., 1959; Martinez-Conde et

Received Dec. 17, 2013; revised July 7, 2014; accepted July 11, 2014.

Author contributions: K.H., M.R., and M.L. designed research; K.H., C.C., M.R., and M.L. performed research; K.H., M.R., and M.L. analyzed data; K.H., C.C., M.R., and M.L. wrote the paper.

This work was supported by Deutsche Forschungsgemeinschaft Grants LA-952/3 and LA-952/6 to M.L. and National Institutes of Health Grants EY18363 and 1127216 to M.R.

The authors declare no competing financial interests.

${ }^{*}$ M.R. and M.L. contributed equally to this work with shared senior authorship.

Correspondence should be addressed to Prof. Dr. Markus Lappe, Institute for Psychology, University of Muenster,

Fliednerstrasse 21, 48149 Muenster, Germany. E-mail: mlappe@uni-muenster.de.

DOI:10.1523/JNEUROSCI.5277-13.2014

Copyright $\odot 2014$ the authors $\quad 0270-6474 / 14 / 3411665-08 \$ 15.00 / 0$ al., 2006). But this hypothesis has remained controversial (Collewijn and Kowler, 2008; Poletti et al., 2010), and an emerging body of literature indicates that microsaccades are controlled movements generated to maintain fixation or acquire visual information according to the task (Hafed et al., 2009; Ko et al., 2010; Engbert et al., 2011; Otero-Millan et al., 2013; Poletti et al., 2013b).

We reasoned that, if microsaccades are part of an active visuomotor process such as saccades, their control should be adaptable. We therefore designed two experiments to investigate whether microsaccades undergo plastic changes similar to those exhibited by saccades during exposure to chronically altered visual input. For large saccades directed toward nonfoveated targets, adaptation is experimentally induced by stepping the target during the saccade to create a systematic visual error (McLaughlin, 1967). Microsaccades can also be target-directed, for example, in response to a small target step (Timberlake et al., 1972). In the first experiment, we studied the adaptation of target-directed microsaccades by using a miniaturized version of the adaptation procedure commonly used for saccades. In the second experiment, we focused on fixational saccades, the microsaccades that occur spontaneously (i.e., without any change in the external stimulus) during fixation on a marker. We show that both types of microsaccades are continually monitored and adapted by the oculomotor system.

\section{Materials and Methods}

Subjects. Six subjects participated in Experiment 1 (3 males and 3 females; average age $33 \pm 5$ years). Two subjects were authors of this study, whereas the others were naive about the purpose of the experiments. Five subjects ( 3 males and 2 females; average age $31 \pm 8$ years) participated in 
Experiment 2. Four of them had also participated in Experiment 1. Informed consent was obtained from all participants following the procedures approved by the Boston University Charles River Campus Institutional Review Board and the Declaration of Helsinki.

Stimuli. The stimulus consisted of a small, high-contrast luminous square ( $3 \operatorname{arcmin}$ by $3 \operatorname{arcmin} ; 52 \mathrm{~cd} / \mathrm{m}^{2}$ ), which we refer to as the target in Experiment 1 and as the fixation marker in Experiment 2. It was displayed on a dark background on a fast phosphor CRT monitor (Iyama HM204DT) at a resolution of $1024 \times 768$ pixels and vertical refresh rate of $100 \mathrm{~Hz}$ in an otherwise completely dark room. A dental imprint bite bar and a head rest prevented movement of the head and kept subjects at a distance of $123 \mathrm{~cm}$ from the monitor. Stimuli were observed monocularly with the right eye while the left eye was patched. Stimuli were rendered by means of EyeRIS, a hardware/software system for gazecontingent display control that enables precise synchronization between eye movement data and the refresh of the image on the monitor (Santini et al., 2007). EyeRIS saved the timing of occurrence of all relevant events in the course of each trial, enabling successive offline analysis of the validity of each trial.

Eye tracking. Movements of the right eye were continuously recorded by means of a Generation 6 Dual Purkinje Image (DPI) Eyetracker (Fourward Technologies). The internal noise of this system is $\sim 20$ arcsec (Crane and Steele, 1985), enabling a spatial resolution of $\sim 1^{\prime}$ (Stevenson and Roorda, 2005). Vertical and horizontal eye positions were sampled at $1 \mathrm{kHz}$ and recorded for subsequent analysis. For the target shift during the experiments, microsaccades were detected online by means of a realtime algorithm for velocity estimation (Santini et al., 2007) and a conservative speed threshold of $10 \%$. For the offline analysis of amplitude changes, a speed $>6 \% \mathrm{~s}$ was used to identify saccades, and their amplitude was then calculated as the distance between the locations in which eye speed became $>2 \%$ s (saccade onset) and that in which it dropped $<2 \%$ (saccade offset). This classification was performed automatically.

Calibration procedure. Every session started with preliminary setup operations that lasted a few minutes. The subject was positioned optimally and comfortably in the apparatus. Subsequently, a calibration procedure was performed in two phases. In the first phase (automatic calibration), subjects sequentially fixated on each of the nine points of a $3 \times 3$ grid, as it is standard in oculomotor experiments. These points were located $2.65^{\circ}$ apart on both the horizontal and vertical axes. In the second phase (manual calibration), subjects confirmed or refined the voltage-to-pixel mapping given by the automatic calibration. In this phase, they fixated again on each of the nine points of the grid while the location of the line of sight estimated on the basis of the automatic calibration was displayed in real time on the screen. Subjects used a joypad to correct the predicted gaze location, if necessary. These corrections were then incorporated into the voltage-to-pixel transformation. This dual-step calibration allows a more accurate localization of gaze position than standard single-step procedures, improving 2D localization of the line of sight by approximately one order of magnitude (Poletti et al., 2013b).

Adaptation of target-directed microsaccades. In Experiment 1, each trial started with the presentation of the target at the center of the CRT display. The subject was instructed to precisely maintain gaze on the target. After a random delay (500-700 ms), the target in the backward adaptation condition shifted to the right along the horizontal axis by $30^{\prime}$. After this first displacement, the target was moved by $15^{\prime}$ to the left as soon as the onset of a microsaccade in the same direction of the first target displacement was detected. Offline data analysis showed that the average delay between microsaccade onset and the shift of the stimulus in the adaptation procedure was $18 \mathrm{~ms}$. The average saccade duration was 34 $\mathrm{ms}$. The target remained at this final position for $500 \mathrm{~ms}$ before being extinguished. The next trial started with the reappearance of the target at the center of the monitor after a dark period of $600 \mathrm{~ms}$. Because the target displacements were small, subjects failed to elicit the saccade on a subset of trials ( $28 \%$ on average). Trials in which the subject did not respond to the initial target displacement with a rightward microsaccade within 400 ms were aborted.

In the forward adaptation condition, the target initially shifted $20^{\prime}$ to the right and then, during the saccade, a further $15^{\prime}$ to the right. Thus, the intrasaccadic step sizes were the same for both adaptation conditions, but the primary amplitude was different. This amplitude difference was necessary to ensure that, for forward adaptation, the total saccade amplitude stayed within the range of microsaccades $\left(20^{\prime}+15^{\prime}=35^{\prime}\right.$ in our case $)$. In backward adaptation, on the other hand, we used a $30^{\prime}$ primary amplitude with a step size of $15^{\prime}$ to ensure that subjects would elicit a saccade even in case of perfect adaptation. Had we used a primary amplitude of $20^{\prime}$, complete adaptation would correspond to a microsaccade of $5^{\prime}$, which most of subjects are not able to elicit.

Each experimental session consisted of 170 adaptation trials. They were preceded by 30 trials in which only the first target step occurred and the target then remained immobile at its new position (no intrasaccadic displacement). These trials served to determine baseline amplitudes before adaptation. At the end of the session, 50 trials with no intrasaccadic step were added to wash out adaptation before the subject left the experiment. Furthermore, to reduce predictability and avoid express saccades, occasionally the target was displaced to the left, rather than to the right and was extinguished during the saccade. These trials were randomly interspersed with the others but were rare ( 36 trials across the session). They were excluded from data analysis. The subject was able to take a rest from the experiment by looking away from the center of the screen or closing the eyes, which induced a waiting period within the experimental session.

To evaluate the effect of the procedure, we compared the amplitudes of the first microsaccades occurring after target displacement in the first 30 trials (the baseline phase) and in the last 100 adaptation trials of the same session. This experimental choice was dictated by the multiple experimental challenges we had to face for measuring adaptive changes at the microscopic level, which prevented us from following the more traditional approaches of a separate postadaptation test phase. An important issue was the considerably larger Fano factor of microsaccades (i.e., the consideration that, for microsaccades, the amplitude variance amounts to a much larger proportion of the mean amplitude than for larger saccades). This increment in variability implies the need for comparing performance over large samples, a problem further amplified by the fact that, because microsaccades already possess small amplitudes, the amplitude changes resulting from adaptation are even smaller and therefore difficult to quantify statistically. We therefore needed a large number of saccades in a long-term stable situation to reliably measure the effect of adaptation. Collection of such large pools of data was, however, not possible in standard postadaptation test phases because the typical aftereffects of saccadic adaptation tend to diminish rather quickly. Although this approach may underestimate the saccadic change, as adaptation may be still occurring in the last 100 trials of each session, our finding of a significant amplitude change relative to the initial baseline demonstrates that target-directed microsaccades are adaptable.

Adaptation of fixational saccades. Microsaccades may also occur spontaneously during sustained fixation. In this study, we refer to these movements as fixational saccades, to distinguish them from the microsaccades elicited by displacement of the fixation marker. The direction of fixational saccades appears to be random (i.e., they are directed away from the fixation point as often as toward it). To induce adaptation of fixational saccades, we displaced the fixation marker based on an online estimate of direction and amplitude of each occurring saccade (Robinson et al., 2003; Havermann and Lappe, 2010).

To collect sufficient data from each individual, we interleaved multiple adaptation and test phases in a single session and compared forward and backward adaptation at different fixation locations within the session (Shelhamer and Clendaniel, 2002; Alahyane and Pélisson, 2004). The characteristics of fixational saccades are known to vary across experimental sessions, probably reflecting the subject's degree of motivation, and averaging across sessions would therefore complicate quantification of the effect. For this reason, we developed a procedure that provided us with an evaluation of saccadic adaptation within a single experimental session. Given that in Experiment 1 we had used a set of saccades from the adaptation phase, in Experiment 2, to demonstrate robustness of the effect with respect to the specific assessment method, we inserted multiple test phases during the same experimental session. This approach enabled us to collect sufficiently large number of saccades to evaluate 
statistical significance. We decided to contrast two opposite adaptation procedures rather than compare the effect of adaptation at one fixation position and no adaptation at the other because adaptation would likely transfer by some degree to the other position, making observation of the effect difficult. By comparing changes, this approach optimized statistical power. This was necessary because fixational saccades are not very frequent and occur in all directions, thus yielding a modest number of repetitions of the adaptation procedure compared with more traditional experiments with larger saccades.

An experimental session, therefore, consisted of successive fixation periods (trials), each of $\sim 12 \mathrm{~s}$ duration, which alternated between two locations of the display: one $75^{\prime}$ above (top location) and one $75^{\prime}$ below (bottom location) the center of the CRT. During each period, an adaptation phase preceded a test phase. The fixation marker appeared at one of the two locations, and the adaptation procedure started after an initial $400 \mathrm{~ms}$ interval allowed for the establishment of fixation. In this phase, every fixational saccade induced a displacement in the position of the fixation marker (50\% of the saccade amplitude) either along (forward adaptation) or against the saccade direction (backward adaptation). The detection of the saccade onset was the same as in Experiment 1, but the estimation of saccade amplitude and direction introduced an additional delay of $48 \mathrm{~ms}$, on average. Following earlier procedures (Havermann and Lappe, 2010), the target was therefore switched off as soon as the saccade onset was detected and was turned on at the appropriate position after the estimation of saccade amplitude and direction was finished. After $8 \mathrm{~s}$ of adaptation, a test phase of $4 \mathrm{~s}$ began, in which the marker did not move. The trial ended with a blank screen $(500 \mathrm{~ms})$. Thereafter, the fixation target appeared at the other monitor position, and the subject performed the manual calibration procedure again for this target before the next trial started.

Fixation periods were grouped in blocks of 30 trials, always starting with fixation at the bottom monitor location. Different adaptation procedures (forward or backward) were conducted at the two locations, so that the intrasaccadic displacement was constant during any fixation period but changed between successive periods. Several fixation blocks were performed in each experimental session (the precise number determined by the subject's status of concentration), with either the forward adaptation procedure (Forward Above condition) or the backward adaptation procedure (Backward Above condition) conducted at the top location. Different conditions were conducted on different days. To ensure adaptation specific to eye position, subjects were required to maintain strict fixation on the marker during each block. If the subject looked away from the fixation marker by $>72^{\prime}$, data collected during the considered fixation period and during the remainder of the block were discarded because an eye-position-specific adaptation could no longer be guaranteed. Between 71 and 356 fixational saccades were collected for each subject, eye position, and condition. To measure adaptation, we compared the median amplitudes of the fixational saccades measured during the test phases at the two locations. To this end, we pooled all fixational saccades following the first one in each test phase.

\section{Results}

\section{Adaptation of target-directed microsaccades}

We first examined whether target-directed saccades with amplitudes within the microsaccade range $\left(30^{\prime}\right)$ could be adapted using the paradigm initially developed by McLaughlin (1967) and commonly used for larger saccades. The subject was instructed to maintain steady gaze on a small $\operatorname{dot}\left(3^{\prime}\right.$ by $3^{\prime}$; the target), which stepped systematically, but at unpredictable times, from its initial position at the center of the CRT display to a very nearby location on the horizontal axis (Fig. 1A). Every time the sudden step of the target was followed by a microsaccade in the same direction, the target shifted again by a fixed amount. This procedure led to adaptive changes in microsaccade amplitude, as shown for an individual subject in Figure $1 B, C$. At the beginning of the experiment (Fig. 1B), when adaptation had not yet occurred, the subject made a saccade toward the new target location, $30^{\prime}$ to the right of the initial fixation position. During this saccade, the dot shifted back $15^{\prime}$ to the left. Approximately 400 ms later, the subject made a corrective saccade to the final dot location at $15^{\prime}$. In contrast, later in the same experiment (Fig. 1C), when adaptation had already started exerting its effect, the subject responded to the dot displacement with a smaller microsaccade of $\sim 20^{\prime}$, which better matched the final location of the target after the back-step. Thus, repeated exposure to an intrasaccadic backward step of the target led to shortening of microsaccade amplitude. A decrease in amplitude was measured in each of the 6 subjects (Fig. 1D). Averaged over all subjects, the microsaccade amplitude decreased by 3.4' (two-tailed paired $t$ test: $p<0.002$; Fig. $1 E$ ), which was equivalent to $23 \%$ of the target shift, a change within the adaptation range of normal saccades (Pélisson et al., 2010). For larger saccades, it is known that amplitude changes do not depend on corrective saccades (Wallman and Fuchs, 1998) and that the execution of corrective saccades and amplitude adaptation are two separate processes for landing error correction (Panouillères et al., 2011). In our experiments, corrective saccades as in Figure $1 B$ occurred not in all subjects, and there was no visible correlation between adaptation state and their occurrence.

A forward intrasaccadic step of the target (i.e., a change in position in the same direction of the microsaccade) led to a lengthening of microsaccade amplitude (Fig. 2). In this case, the target shifted to the right first by $20^{\prime}$ and then by an additional $15^{\prime}$ during the occurrence of the microsaccade (Fig. 2A). Before adaptation, the subject responded to the two target steps with two successive microsaccades, the first one of $\sim 20^{\prime}$ (Fig. $2 B$ ). Later, after many adaptation trials, a single larger microsaccade, which ended closer to the final target location, was performed (Fig. 2C). Forward adaptation resulted in an increase of amplitude in 5 of 6 subjects (Fig. 2D), with an average increase of $2.7^{\prime}$ (i.e., $17.8 \%$ of the target shift; Fig. $2 E$; two-tailed paired $t$ test: $p<0.037)$.

The results from Experiment 1 show that target-directed microsaccades of a few minutes of arc can be adapted in amplitude much like normal saccades of several degrees in size. Their amplitude adjusts over time when a minute intrasaccadic displacement of the target occurs. Plasticity at this microscopic scale is surprising: saccadic adaptation is commonly thought to counteract reductions in visual performance caused by the displacement of the target away from the fovea. This explanation does not hold with the adaptation of microsaccades because the target remains within the foveal region at all times.

\section{Adaptation of fixational saccades}

Having shown that the oculomotor system is capable of plastically tuning microscopic saccades, we examined, in a second experiment, whether fixational saccades, the involuntary microsaccades that occur while attempting to maintain precise fixation on a stationary marker, also adapt.

Fixational saccades pose several challenges to the standard adaptation paradigm because they occur unpredictably, and their characteristics cannot be known in advance. Therefore, we estimated the direction and amplitude of a fixational saccade during its occurrence (Robinson et al., 2003; Havermann and Lappe, 2010) and displaced the fixation marker on the basis of these two parameters. That is, we simultaneously applied the adaptation procedure in all saccadic directions and examined whether this approach leads to a global adjustment in the amplitude of fixational saccades (Rolfs et al., 2010). Because the characteristics of fixational saccades vary widely across individuals, this strategy exposed different subjects to different intrasaccadic displacements. Thus, to facilitate detection of possible changes in the 


\section{A}

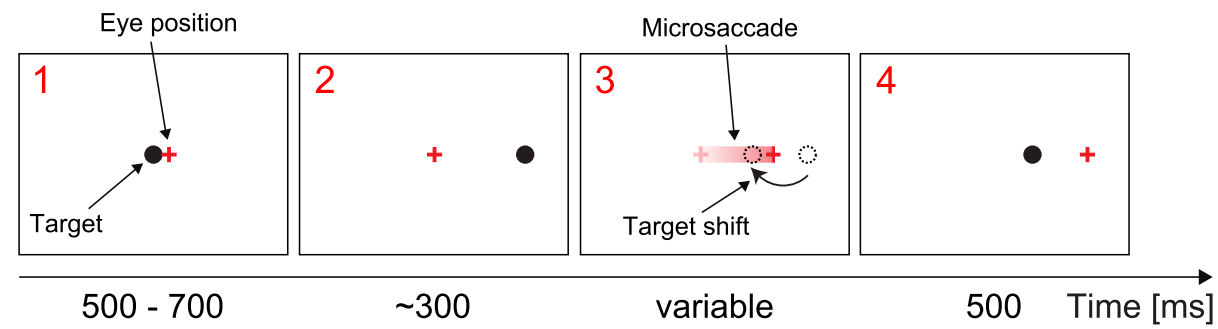

B

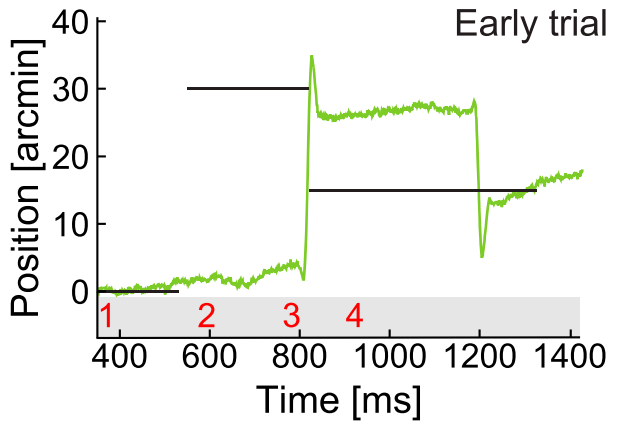

C

D

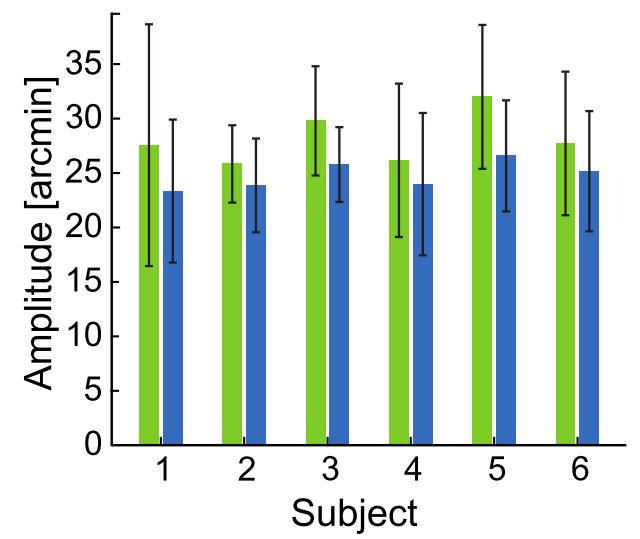

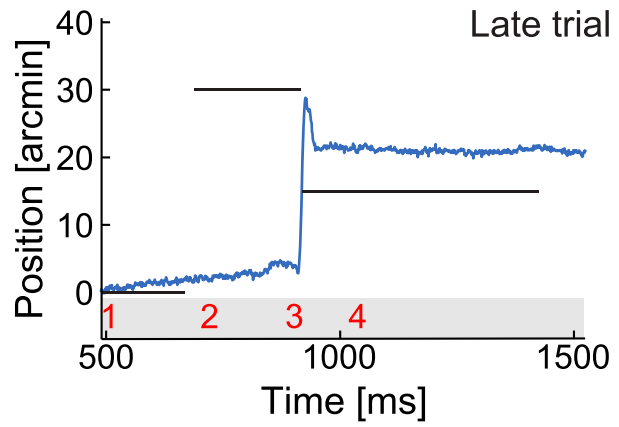

E

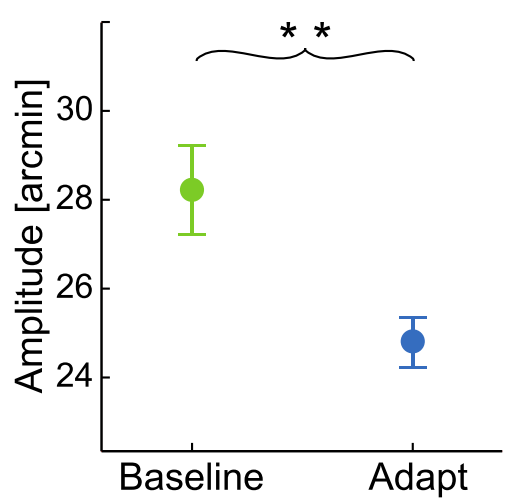

Figure 1. Inward adaptation of target-directed microsaccades. $\boldsymbol{A}$, Experimental procedure. The observer initially fixated the central dot target (1). After a random delay of $500-700 \mathrm{~ms}$, the dot was displaced 30 arcmin to the right (2). The eye followed with a typical saccadic reaction time. The onset of the saccade triggered a backward shift of the target by 15 arcmin ( 3 ), so that gaze was initially not on target after the saccade (4). B, C, Example traces of horizontal eye position. Horizontal lines indicate the position of the target. Numbers on the bottom indicate the corresponding experimental phases in A. D, Mean saccade amplitudes and SDs before (green) and after (blue) saccadic adaptation for each of the six participating subjects. $\boldsymbol{E}$, Means across subjects. Error bars indicate SEM. ${ }^{* *} p<0.01$ (one-sample two-tailed $t$ test).

statistics of fixational saccades, we directly compared the effects of forward and backward adaptation for individual subjects within a single session at different fixation locations (Shelhamer and Clendaniel, 2002; Alahyane and Pélisson, 2004). To collect sufficient numbers of fixational saccades, each session contained multiple short periods of fixation (test phases) interspersed with adaptation phases to establish and refresh adaptation.

Subjects thus alternated prolonged fixation between two different locations, $2.5^{\circ}$ apart, one above and one below the horizontal midline of the display. At each location, they maintained fixation on the marker while an adaptation period $(8 \mathrm{~s})$ preceded a test phase (4s). In the adaptation phase, the fixation marker moved on the display with every fixational saccade, shifting along the saccade direction by $50 \%$ of its amplitude (Fig. $3 A$ ). In the test phase, which served to collect microsaccades during stable fixation, the marker remained stationary on the display. Potential deadaptation that may occur during the test period might reduce the magnitude of the measured adaptation effect. Therefore, we kept these fixation intervals short and refreshed adaptation after each test phase. To eliminate the possibility of spatial biases, different adaptation procedures were conducted at the two fixation locations in separate sessions. In half of the sessions, the marker moved in the direction of the saccade (forward adaptation) at the bottom location and in the direction opposite to that of the saccade (backward adaptation) at the upper fixation location (Fig. $3 B$, Backward Above condition). Displacement directions were inverted in the remaining sessions conducted on different days. Importantly, at both locations, a consistent relationship between fixational saccades and the directions of intrasaccadic displacements was maintained throughout the experimental session, allowing us to directly compare the consequences of prolonged exposure to forward and backward steps of the fixation marker.

In the Backward Above condition, fixational saccades were smaller at the upper fixation position, where the backward adapta- 


\section{A}

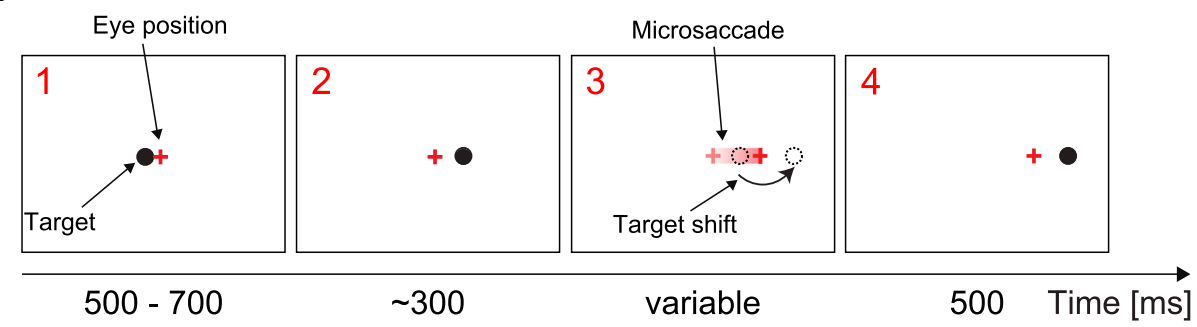

B

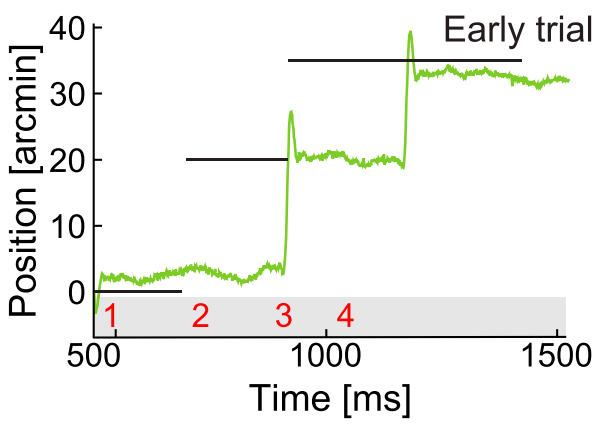

D

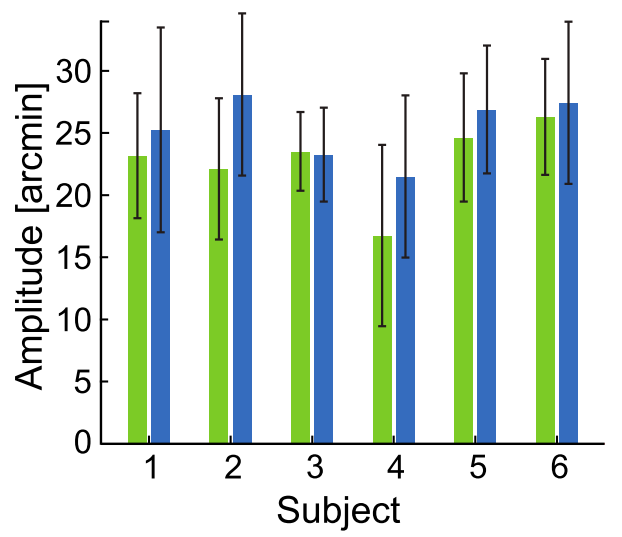

C

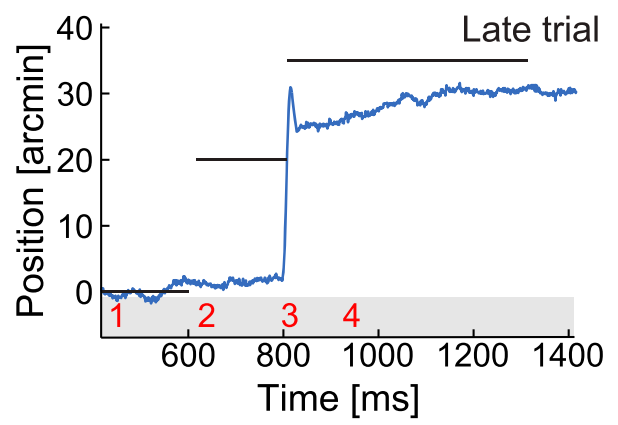

E

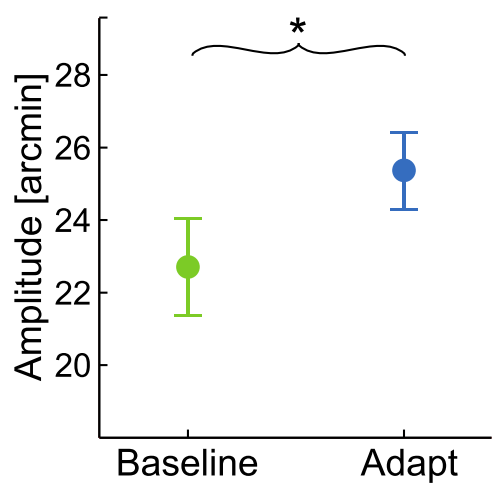

Figure 2. Outward adaptation of target-directed microsaccades. $A$, Experimental procedure. The observer initially fixated the central dot target (1). After a random delay of $500-700 \mathrm{~ms}$, the dot was displaced 20 arcmin to the right (2). During the saccade, the target was shifted by 15 arcmin to the right (3) such that the target was to the right of the gaze direction after the saccade (4). $\boldsymbol{B}$, C, Example traces of horizontal eye position. $\boldsymbol{D}$, Mean saccade amplitudes and SDs before (green) and after (blue) saccadic adaptation for each of the six participating subjects. $\boldsymbol{E}$, Means across subjects. Error bars indicate SEM. ${ }^{*} p<0.05$ (one-sample two-tailed $t$ test).

tion procedure was implemented, than at the lower one, where forward adaptation occurred (Fig. $3 D$; paired two-tailed $t$ test, $p=$ 0.028). A similar amplitude difference but with opposite sign was observed in the Forward Above condition, in which the locations of the two adaptation procedures were exchanged. In this case, fixational saccades were larger at the upper fixation position $(p=0.015)$.

In agreement with previous reports (Cherici et al., 2012), noticeable idiosyncratic variability existed in the amplitude distribution of fixational saccades, so that the mean amplitude varied across observers. As a consequence, because the intrasaccadic target step was proportional to the saccade amplitude, subjects with larger fixational saccades were also exposed, on average, to larger target displacements. This allowed us to examine how adaptation varied with the size of the intrasaccadic shift. Across individuals, the amount of adaptation (i.e., the differences in fixational saccade amplitudes measured following the backward and forward adaptation procedures) was highly correlated with the mean individual amplitude of fixational saccades $\left(r^{2}=0.979\right.$, $p<0.01$; Fig. $3 E)$. That is, all subjects consistently modified their fixational saccades amplitude by the same fraction of the intrasaccadic target step they experienced. We can therefore safely conclude that the observed changes in fixational saccade amplitudes were the result of displacing the target.

\section{Discussion}

Our results demonstrate that oculomotor plasticity extends to an extremely fine spatial scale. Both target-directed microsaccades and fixational saccades are continually monitored and adjusted if the stimulus shifts systematically on the retina during the movement. Oculomotor control at this microscopic level is surprising and is not predicted by mainstream hypotheses about the roles of microsaccades to prevent image fading (Ditchburn et al., 1959; Martinez-Conde et al., 2006) and/or to reflect shifts in covert attention (Hafed and Clark, 2002; Engbert and Kliegl, 2003). It has important implications for the understanding of both sensory processing and oculomotor control.

What is the need for adaptively controlling microsaccades? An interesting possibility, suggested by the results of Experiment 1, is 
A

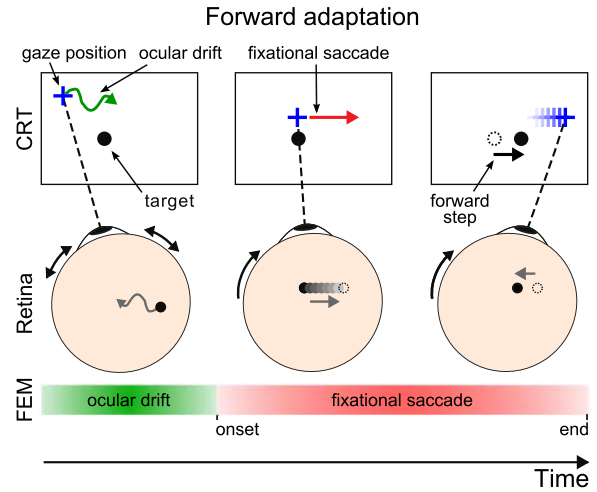

B
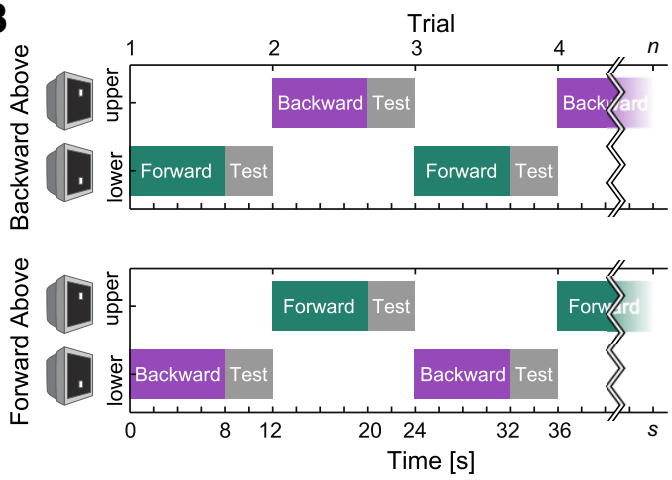

C

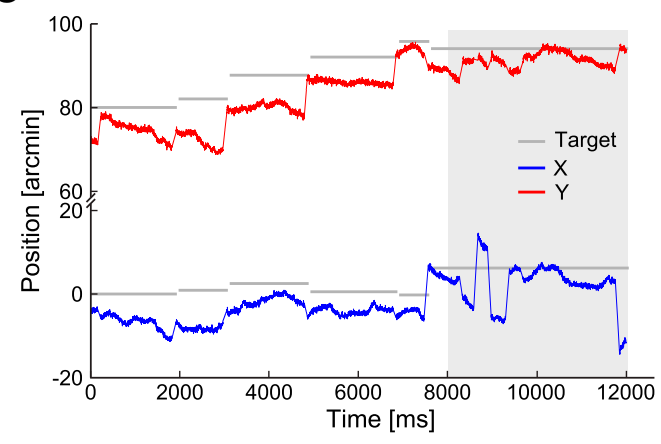

D

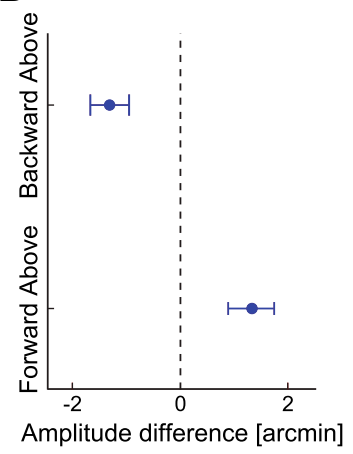

E

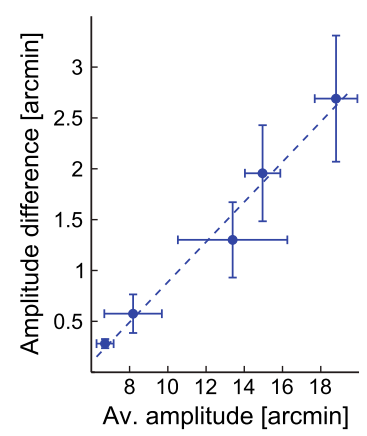

Figure 3. Adaptation of fixational saccades. $A, B$, Experimental procedure. Subjects maintained fixation on a small marker (black dot). Whenever a fixational saccade occurred, the marker shifted by $50 \%$ of the saccade amplitude along the saccade direction (forward adaptation). In the backward adaptation condition (data not shown), the shift was instead against saccade direction. $\boldsymbol{B}$, Subjects alternated periods of fixation (12 s) between two locations, one above and one below the CRT midline, at which different adaptation procedures (forward or backward) were conducted. $C$, Example trace of fixational eye movements showing horizontal (blue) and vertical (red) eye movement components and the intrasaccadic target shifts (gray lines) after establishing fixation in the adaptation phase. Gray area represents the subsequent test phase during which the target did not shift. $\boldsymbol{D}$, Differences in the median amplitudes of fixational saccades at the two fixation locations. $\boldsymbol{E}$, Correlation between the absolute amount of adaptation (averaged across the two conditions) and the mean saccade amplitude of each subject. Subjects with larger fixational saccades exhibited larger changes as a result of adaptation $(p<0.01) . \boldsymbol{D}, \boldsymbol{E}$, Error bars indicate SEM.

that adaptation at this scale serves the same function proposed for larger saccades: ensuring accurate relocations of gaze. Contrary to common assumption, vision is not uniform within the central fovea: cone density declines sharply outside a maximum density region of a few squared arc minutes (Curcio et al., 1990), and it is known that discrimination of fine spatial patterns outside this tiny region is suboptimal in the absence of precisely directed microsaccades even if stimuli fall within the foveola (Poletti et al., 2013 b). Thus, adaptive control of microsaccades may be needed to ensure optimal examination of fine spatial detail with the retinal locus best suited for the task in the same way that saccadic adaptation ensures efficient acquisition of targets outside the fovea.

The results of Experiment 2, however, suggest an additional function of saccadic adaptation to ensuring precise motor control. During sustained fixation, fixational saccades occur in many directions, supposedly serving the function of maintaining the gaze close to the target (Engbert et al., 2011). Fixational saccades tend to move the preferred retinal locus of fixation as often away from the target as toward it (Nachmias, 1959; Kowler and Steinman, 1980). Our intrasaccadic target shifts were always consistent with the saccade direction. Therefore, they affected target location in both ways, sometimes shifting the target closer to the locus of fixation and sometimes further away. Thus, the observed change in amplitude did not have any direct consequence on the accuracy of fixation: the mean fixation error, defined as the Euclidean distance between the microsaccadic end position and the target position, was not different between the forward and the backward adaptation conditions $(p>0.7)$. Why did saccadic adaptation nevertheless occur?

An interesting speculation is that, in addition to ensuring accurate motor behavior, saccadic adaptation plays an important role in the establishment of spatial representations. Saccadic adaptation is known to rely on an extraretinal signal, an internal copy of the motor command known as corollary discharge or efference copy, to predict the retinal position of the target after the saccade (Bahcall and Kowler, 2000; Wong and Shelhamer, 2011; Collins and Wallman, 2012; Herman et al., 2013). A large body of evidence indicates that such corollary discharge signals are also important for establishing stable spatial representations across saccades (Ross et al., 2001; Sommer and Wurtz, 2002; Hamker et al., 2008; Cavanagh et al., 2010; Hafed, 2013; Poletti et al., 2013a), perhaps even spatiotopic ones, as has been recently shown for large saccade adaptation (Zimmermann et al., 2011). In addition to creating the need for motor tuning, the stimulus manipulations present during saccadic adaptation experiments also affect perceptual representations, as they cause systematic mismatches between the postsaccadic retinal inputs and their presaccadic motor predictions. Indeed, for large saccades, the localization of targets presented before the saccade but during adaptation is misperceived, demonstrating a strong correlation between gaze controlling mechanisms and perceptual localization (Bahcall and Kowler, 2000; Awater et al., 2005; Collins et al., 2007). Similar mislocalization might be induced by adaptation of fixational saccades. The adaptation of both saccades and microsaccades may therefore serve to bring retinal and extraretinal 
information in register to ensure the establishment of consistent visuomotor representations of space across eye movements (Zimmermann and Lappe, 2010). In this view, possible corollary discharges associated with microsaccades would help encoding spatial relationships during fixation by ensuring that the postsaccadic location of the fixation target matches the internally generated expectation independent of whether or not it falls within the preferred retinal locus of fixation.

Our results provide further evidence that, under natural viewing conditions, the traditional distinction between saccades and microsaccades may be unjustified (Ko et al., 2010). Microsaccades and saccades have been commonly regarded as separate categories of eye movements because of two primary reasons: unlike saccades, (1) microsaccades tend to occur involuntarily and (2) microsaccades appear unnecessary for the acquisition of visual information as they keep the stimulus within the region of highest acuity at all times. But both considerations have raised doubts. First, like larger saccades, microsaccades can be voluntarily elicited (Haddad and Steinman, 1973) (see our Experiment 1) as well as suppressed (Steinman et al., 1967). Second, as noted above, high-acuity vision drops with eccentricity already within the foveola, so that even microscopic relocations of gaze are helpful in high-acuity tasks (Poletti et al., 2013b).

Indeed, multiple long-standing considerations unify microsaccades and saccades. For example, it has long been known that the distribution of saccadic amplitudes is continuous, with no clear separation between the two types of movements, making the very definition of microsaccades problematic (Collewijn and Kowler, 2008). This amplitude distribution also scales with the size of the scene (von Wartburg et al., 2007; Otero-Millan et al., 2013), suggesting that microscopic and macroscopic saccades serve similar functions, as originally proposed by Cunitz and Steinman (1969) more than half a century ago. Furthermore, microsaccades and saccades are associated with similar extraretinal phenomena (Hafed, 2013) and exhibit highly similar dynamic characteristics (Zuber et al., 1965), an observation that led these authors to first suggest that the two movements share the same control structures (see also Haddad and Steinman, 1973) This proposal has now found experimental support both at the level of brainstem omnipause neurons (Van Gisbergen et al., 1981) and the superior colliculus (Hafed et al., 2009).

Much confusion about the functions of microsaccades seems to have been caused by the relatively large uncertainty in gaze localization, which results from the standard calibration procedure used in oculomotor research. For establishing a correspondence between eyetracker output signals and spatial coordinates, experiments are often preceded by a calibration in which the observer sequentially maintains steady fixation on a number of predetermined points. However, because of the continual occurrence of fixational eye movements, this procedure unavoidably yields uncertainty in the absolute localization of the line of sight: the line projecting to the center of the preferred retinal locus of fixation. The resulting region of uncertainty is as large as the size of the foveola (Cherici et al., 2012) and has traditionally prevented precise determination of where microsaccades shift gaze in the scene. The gaze-contingent calibration procedure used in our experiments improves gaze localization by one order of magnitude over the standard methods. Use of this technique in natural high-acuity tasks has already shown that microsaccades precisely move the preferred retinal locus of fixation to interesting locations in the scene according to the ongoing demands of the task (Ko et al., 2010), improving fine spatial judgments (Poletti et al., 2013b). These results provide strong evidence that microsaccades, like larger saccades, serve an explorative function. The present study adds to this body of literature by showing that plasticity does occur at the microscopic level and that microsaccades and saccades also share similar adaptive strategies.

The neural substrate of the plastic changes observed in this study may be the same one proposed for large saccades. An identified pathway for corollary discharge signals originates in the superior colliculus (Sommer and Wurtz, 2002), a central station in the generation of saccades. Saccade target information is represented as a motor vector in the population activity of the collicular oculomotor map with the rostral part of the colliculus active during fixation (Munoz and Wurtz, 1993). Some neurons in the rostral colliculus show microsaccade-related activity similar to that in the oculomotor map in the caudal colliculus (Hafed et al., 2009) and might provide a corollary discharge during fixational saccades, which can be used to predict the postsaccadic target location. During saccadic adaptation, the saccade command from the colliculus undergoes modification in the cerebellum, where neural activity changes in the oculomotor vermis (Catz et al., 2005, 2008), and the caudal fastigial nucleus appears responsible for tuning saccade amplitude (Scudder and McGee, 2003; Soetedjo and Fuchs, 2006). It is already known that inactivation of the fastigial oculomotor region also affects the locus of fixation and the amplitude of fixational saccades (Guerrasio et al., 2010). Thus, the cerebellum might tune microsaccade commands from the rostral colliculus in the same way it adjusts caudally encoded saccade commands. Further work is needed to test this hypothesis.

\section{References}

Alahyane N, Pélisson D (2004) Eye position specificity of saccadic adaptation. Invest Ophthalmol Vis Sci 45:123-130. CrossRef Medline

Awater H, Burr D, Lappe M, Morrone MC, Goldberg ME (2005) Effect of saccadic adaptation on localization of visual targets. J Neurophysiol 93: 3605-3614. CrossRef Medline

Bahcall DO, Kowler E (2000) The control of saccadic adaptation: implications for the scanning of natural visual scenes. Vision Res 40:2779-2796. CrossRef Medline

Barlow HB (1952) Eye movements during fixation. J Physiol 116:290-306. Medline

Catz N, Dicke PW, Thier P (2005) Cerebellar complex spike firing is suitable to induce as well as to stabilize motor learning. Curr Biol 15:2179-2189. CrossRef Medline

Catz N, Dicke PW, Thier P (2008) Cerebellar-dependent motor learning is based on pruning a purkinje cell population response. Proc Natl Acad Sci U S A 105:7309-7314. CrossRef Medline

Cavanagh P, Hunt AR, Afraz A, Rolfs M (2010) Visual stability based on remapping of attention pointers. Trends Cogn Sci 14:147-153. CrossRef Medline

Cherici C, Kuang X, Poletti M, Rucci M (2012) Precision of sustained fixation in trained and untrained observers. J Vis 12:1-16. CrossRef Medline

Collewijn H, Kowler E (2008) The significance of microsaccades for vision and oculomotor control. J Vis 8:20-21. CrossRef Medline

Collins T, Wallman J (2012) The relative importance of retinal error and prediction in saccadic adaptation. J Neurophysiol 107:3342-3348. CrossRef Medline

Collins T, Vergilino-Perez D, Beauvillain C, Doré-Mazars K (2007) Saccadic adaptation depends on object selection: evidence from between- and within-object saccadic eye movements. Brain Res 1152:95-105. CrossRef Medline

Crane HD, Steele CM (1985) Generation-V dual-Purkinje-image eyetracker. Appl Optics 24:527-537. CrossRef Medline

Cunitz RJ, Steinman RM (1969) Comparison of saccadic eye movements during fixation and reading. Vision Res 9:683-693. CrossRef Medline

Curcio CA, Sloan KR, Kalina RE, Hendrickson AE (1990) Human photoreceptor topography. J Comp Neurol 292:497-523. CrossRef Medline

Ditchburn RW, Fender DH, Mayne S (1959) Vision with controlled movements of the retinal image. J Physiol 145:98-107. Medline 
Engbert R, Kliegl R (2003) Microsaccades uncover the orientation of covert attention. Vision Res 43:1035-1045. CrossRef Medline

Engbert R, Mergenthaler K, Sinn P, Pikovsky A (2011) An integrated model of fixational eye movements and microsaccades. Proc Natl Acad Sci U S A 108:E765-E770. CrossRef Medline

Guerrasio L, Quinet J, Büttner U, Goffart L (2010) Fastigial oculomotor region and the control of foveation during fixation. J Neurophysiol 103: 1988-2001. CrossRef Medline

Haddad GM, Steinman RM (1973) The smallest voluntary saccade: implications for fixation. Vis Res 13:298-300. Medline

Hafed ZM (2013) Alteration of visual perception prior to microsaccades. Neuron 77:775-786. CrossRef Medline

Hafed ZM, Clark JJ (2002) Microsaccades as an overt measure of covert attention shifts. Vision Res 42:2533-2545. CrossRef Medline

Hafed ZM, Goffart L, Krauzlis RJ (2009) A neural mechanism for microsaccade generation in the primate superior colliculus. Science 323:940-943. CrossRef Medline

Hamker FH, Zirnsak M, Calow D, Lappe M (2008) The peri-saccadic perception of objects and space. PLoS Comput Biol 4:e31. CrossRef Medline

Havermann K, Lappe M (2010) The influence of the consistency of postsaccadic visual errors on saccadic adaptation. J Neurophysiol 103:33023310. CrossRef Medline

Herman JP, Blangero A, Madelain L, Khan A, Harwood MR (2013) Saccade adaptation as a model of flexible and general motor learning. Exp Eye Res 114:6-15. CrossRef Medline

Ko HK, Poletti M, Rucci M (2010) Microsaccades precisely relocate gaze in a high visual acuity task. Nat Neurosci 13:1549-1553. CrossRef Medline

Kowler E, Steinman RM (1980) Small saccades serve no useful purpose: reply to a letter by R. W. Ditchburn. Vision Res 20:273-276. CrossRef Medline

Martinez-Conde S, Macknik SL, Troncoso XG, Dyar TA (2006) Microsaccades counteract visual fading during fixation. Neuron 49:297-305. CrossRef Medline

McLaughlin S (1967) Parametric adjustment in saccadic eye movements. Percept Psychophys 2:359-362. CrossRef

Munoz DP, Wurtz RH (1993) Fixation cells in monkey superior colliculus: characteristics of cell discharge. J Neurophysiol 70:559-575. Medline

Nachmias J (1959) Two-dimensional motion of the retinal image during monocular fixation. J Opt Soc Am 49:901-908. CrossRef Medline

Otero-Millan J, Macknik SL, Langston RE, Martinez-Conde S (2013) An oculomotor continuum from exploration to fixation. Proc Natl Acad Sci U S A 110:6175-6180. CrossRef Medline

Panouillères M, Urquizar C, Salemme R, Pélisson D (2011) Sensory processing of motor inaccuracy depends on previously performed movement and on subsequent motor corrections: a study of the saccadic system. PLoS One 6:e17329. CrossRef Medline

Pélisson D, Alahyane N, Panouillères M, Tilikete C (2010) Sensorimotor adaptation of saccadic eye movements. Neurosci Biobehav Rev 34:11031120. CrossRef Medline

Poletti M, Listorti C, Rucci M (2010) Stability of the visual world during eye drift. J Neurosci 30:11143-11150. CrossRef Medline

Poletti M, Burr DC, Rucci M (2013a) Optimal multimodal integration in spatial localization. J Neurosci 33:14259-14268. CrossRef Medline

Poletti M, Listorti C, Rucci M (2013b) Microscopic eye movements com- pensate for nonhomogeneous vision within the fovea. Curr Biol 23:16911695. CrossRef Medline

Robinson FR, Noto CT, Bevans SE (2003) Effect of visual error size on saccade adaption in monkey. J Neurosci 90:1235-1244. Medline

Rolfs M (2009) Microsaccades: small steps on a long way. Vision Res 49: 2415-2441. CrossRef Medline

Rolfs M, Knapen T, Cavanagh P (2010) Global saccadic adaptation. Vision Res 50:1882-1890. CrossRef Medline

Ross J, Morrone MC, Goldberg ME, Burr DC (2001) Changes in visual perception at the time of saccades. Trends Neurosci 24:113-121. CrossRef Medline

Santini F, Redner G, Iovin R, Rucci M (2007) EyeRIS: a general-purpose system for eye-movement-contingent display control. Behav Res Methods 39:350-364. CrossRef Medline

Scudder CA, McGee DM (2003) Adaptive modification of saccade size produces correlated changes in the discharges of fastigial nucleus neurons. J Neurophysiol 90:1011-1026. CrossRef Medline

Shelhamer M, Clendaniel RA (2002) Context-specific adaptation of saccade gain. Exp Brain Res 146:441-450. CrossRef Medline

Soetedjo R, Fuchs AF (2006) Complex spike activity of purkinje cells in the oculomotor vermis during behavioral adaptation of monkey saccades. J Neurosci 26:7741-7755. CrossRef Medline

Sommer MA, Wurtz RH (2002) A pathway in primate brain for internal monitoring of movements. Science 296:1480-1482. CrossRef Medline

Steinman RM, Haddad GM, Skavenski AA, Wyman D (1973) Miniature eye movement. Science 181:810-819. CrossRef Medline

Steinman RM, Cunitz RJ, Timberlake GT, Herman M (1967) Voluntary control of microsaccades during maintained monocular fixation. Science 155:1577-1579. CrossRef Medline

Stevenson SB, Roorda A (2005) Miniature eye movements measured simultaneously with ophthalmic imaging and a Dual-Purkinje image eye tracker. J Vis 5:590.

Timberlake GT, Wyman D, Skavenski AA, Steinman RM (1972) The oculomotor error signal in the fovea. Vision Res 12:1059-1064. CrossRef Medline

Van Gisbergen JA, Robinson DA, Gielen S (1981) A quantitative analysis of generation of saccadic eye movements by burst neurons. J Neurophysiol 45:417-442. Medline

von Wartburg R, Wurtz P, Pflugshaupt T, Nyffeler T, Lüthi M, Müri RM (2007) Size matters: saccades during scene perception. Perception 36: 355-365. CrossRef Medline

Wallman J, Fuchs AF (1998) Saccadic gain modification: visual error drives motor adaptation. J Neurophysiol 80:2405-2416. Medline

Wong AL, Shelhamer M (2011) Sensorimotor adaptation error signals are derived from realistic predictions of movement outcomes. J Neurophysiol 105:1130-1140. CrossRef Medline

Zimmermann E, Burr D, Morrone MC (2011) Spatiotopic visual maps revealed by saccadic adaptation in humans. Curr Biol 21:1380-1384. CrossRef Medline

Zimmermann E, Lappe M (2010) Motor signals in visual localization. J Vis 10:2.1-11. CrossRef Medline

Zuber BL, Stark L, Cook G (1965) Microsaccades and the velocityamplitude relationship for saccadic eye movements. Science 150:14591460. CrossRef Medline 